\title{
Change in Taste Preference to Capsaicin and Catechin Due to Aging in Mice
}

\author{
Masataka NARUKAWA $^{1,2}$ and Takumi MISAKA ${ }^{1}$ \\ ${ }^{1}$ Department of Applied Biological Chemistry, Graduate School of Agricultural and Life Sciences, \\ The University of Tokyo, Yayoi 1-1-1, Bunkyo-ku, Tokyo 113-8657, Japan \\ ${ }^{2}$ Department of Food and Nutrition, Kyoto Women's University, 35 Kitahiyoshi-cho, Imakumano, \\ Higashiyama-ku, Kyoto 605-8501, Japan \\ (Received December 14, 2020)
}

\begin{abstract}
Summary Taste is a chemical sensation that primarily detects nutrients present in food, and maintenance of taste sensations is important for ensuring that older people have a balanced nutritional diet. While several reports have suggested that taste sensitivity changes with age, the molecular mechanisms underlying this phenomenon are still unclear. Previous studies on the matter have focused mainly on the relationship between aging and taste detection of specific basic taste-inducing substances, and other than for these basic substances, understanding of how aging affects the detection of taste is limited. Therefore, to understand the effect that aging has on the taste detection of some familiar substances found in our daily meals, namely capsaicin and catechin, we investigated age-related changes in taste preferences to capsaicin and catechin in young and old C57BL/6J mice using a 48-h two-bottle preference test. For the capsaicin stimuli, the mice showed avoidance behavior in a concentration-dependent manner. However, we observed that there was no significant difference in the preference ratio for capsaicin between young and old mice. For the catechin stimuli, although both age groups showed avoidance behavior in a concentration-dependent manner, the preference ratio in old mice showed significantly higher values than those in young mice. This suggests that catechin sensitivity is declined due to aging. Thus, we observed that catechin sensitivity decreases with age, but capsaicin sensitivity does not.
\end{abstract}

Key Words aging, taste, taste preference, capsaicin, catechin

Among older people, health has become an important social issue due to the progressively aging population in most developed countries. A balanced and nutritional diet is imperative for maintaining good health. Taste is a chemical sensation that primarily detects nutrients present in food, and maintenance of taste sensations is important for ensuring that older people have a balanced nutritional diet.

The taste of food consists of various qualities. Physiologically, the taste is classified into five basic categories, such as salty, sweet, bitter, sour, and umami. Although pungency and astringency are not classified into the basic taste categories, they play important roles in tasting certain foods.

Several reports have suggested that taste sensitivity changes with age (1-5). However, the molecular mechanisms underlying this phenomenon are unclear. Previous studies focused mainly on the relationship between aging and the taste detection of specific basic taste-inducing substances, such as sodium chloride (salty), citric acid (sour), denatonium benzoate (bitter), sucrose (sweet), and monosodium glutamate (umami). It is still poorly understood how aging affects the detection of taste substances other than for these specific basic

E-mail: narukawa@kyoto-wu.ac.jp taste-inducing substances. An investigation into the effects of aging on taste detection of substances other than specific basic taste-inducing substances may be useful for clarifying the underlying mechanisms involved in the age-dependent changes of taste sensitivity.

We chose to use capsaicin and catechin to better understand the effect aging has on the taste detection of familiar substances found in our daily meals. Capsaicin is a representative pungent component found in hot peppers. Catechin is a representative polyphenol found in tea and wine. In this study, we investigated the age-related changes in taste preference to such components, using young and old mice.

\section{MATERIALS AND METHODS}

Materials. Capsaicin and denatonium benzoate (denatonium) were purchased from Sigma (St. Louis, MO). Catechin was kindly supplied as Sunphenon 90LBOP from Taiyo Chemical Co., Ltd. (Mie, Japan). The Sunphenon 90LB-OP is a catechin mixture and contains $85.5 \%$ catechins (epigallocatechin gallate (EGCg) 46\%, epigallocatechin (EGC) 17.3\%, epicatechin (EC) 8.2\%, epicatechin gallate (ECg) $8.2 \%$, gallocatechin 2.7\%, gallocatechin gallate $1.6 \%$, and catechin $1.5 \%$ ) and $0.8 \%$ caffeine. All other reagents were of analytical grade and 
were obtained from standard suppliers.

Animals. The study cohort comprised male C57BL/6J (B6) mice (CLEA Japan, Inc., Tokyo, Japan). The B6 strain has previously been used in research involving taste science. We divided the mice into two age groups: young mice aged $8-12$ wk (body weight: $23.7 \pm 0.6 \mathrm{~g}$ at $8 \mathrm{wk}$ ) and old mice aged $124-128 \mathrm{wk}$ (body weight: $29.6 \pm 1.0 \mathrm{~g}$ at $124 \mathrm{wk}$ ). The young and old mice were housed at the CLEA Japan Animal Care Facility until the age of 7 and 123 wk, respectively. Thereafter, they were simultaneously moved to the University of Tokyo Animal Care Facility. They had ad libitum access to a standard laboratory solid food (Rodent Diet CE-2; CLEA Japan) and distilled water. The surrounding temperature and humidity were maintained at $23^{\circ} \mathrm{C}$ and $55 \%$, respectively, with a $12-\mathrm{h} / 12$-h light/ dark cycle (lights switched on at $0800 \mathrm{~h}$ ). Mice with normal shapes and feeding behaviors were used. All experiments were performed in accordance with the protocols approved by The University of Tokyo Animal Care Committee (Approval Number: P18-075).

Initially, mice were subjected to a grip strength test, followed by a 48-h two-bottle preference test. The behavioral assays for the young and old mice were performed simultaneously, and afterwards the trigeminal ganglion (TG) was collected from all mice.

Grip strength test. Grip strength of the forelimb was measured using a Grip Strength Meter (GPM-101B; Melquest, Toyama, Japan). As the mouse grasped the bar, the peak pull force in grams was recorded on a digital force transducer. Measurements were repeated five times. Excluding the maximum and minimum values, the average of the three middle values was calculated as the grip strength.

Forty-eight-hour two-bottle preference test. Young mice $(n=6)$ and old mice $(n=5-6)$ were caged individually and given $48 \mathrm{~h}$ of access to two bottles, one containing deionized water and the other containing a tastant solution. After $24 \mathrm{~h}$, the bottle positions were switched to avoid positional effects. The ratio of tastant volume to total liquid consumed was recorded. The preference ratios of the tastants were calculated as follows: tastant intake/total fluid intake (tastant intake + water intake). The tastant solutions for the two-bottle preference test were as follows: $0.3 \mathrm{mM}$ denatonium, $0.1-3 \mu \mathrm{M}$ capsaicin, and $0.003-0.1 \%$ catechin. The tested concentrations were used in ranges, where the preference ratios changed in a concentration-dependent manner. The capsaicin solution was used by diluting the ethyl alcohol (EtOH) stock solution 10,000 times. When measuring the preference for capsaicin, EtOH was added to water (final concentration $=0.01 \%$ ). The tastant solutions were presented in an ascending series of concentrations. Mice were habituated by presenting two bottles that contained water for only $1 \mathrm{wk}$ before starting the preference test, and it was confirmed that the mice drank water evenly from both bottles containing water. Apart from mice that died during the preference test, each mouse was tested using all solutions.

Real-time RT-PCR. After the behavioral assays, the
A

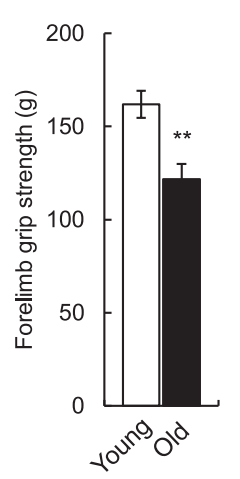

$B$

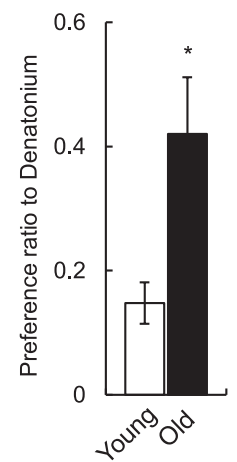

Fig. 1. Comparisons of (A) forelimb grip strength and (B) preference ratio to bitter taste between young (white bars, $n=6$ ) and old mice (black bars, $n=6$ ). * and ** indicate $p<0.05$ and 0.01 , respectively (Welch's $t$-test).

young $(n=6)$ and old mice $(n=5)$ were sacrificed by cervical dislocation, and their TGs were removed. Cervical dislocation has performed by an operator who mastered technical skills to ensure that loss of consciousness is rapidly induced. Total RNA from the TGs was extracted using RNeasy Mini Columns (Qiagen, Venlo, Netherlands). Genomic DNA digestion was performed using an RNase-free DNase Set (Qiagen). First-strand cDNA was generated from total RNA by reverse transcription (Superscript IV Reverse Transcription Kit; Life Technologies, Gaithersburg, MD). The mRNA transcript levels were determined by quantitative polymerase chain reaction (qPCR; ABI Prism 7000 Sequence Detection System; Thermo Fisher Scientific). PCR amplification was performed using the SYBR green PCR system (Thermo Fisher Scientific). qPCR primers were designed as follows: transient receptor potential vanilloid 1 (Trpv1): forward primer, 5'-TTCCTGCAGAAGAGCAAGAAGC-3'; reverse primer, 5'-CCCATTGTGCAGATTGAGCAT-3'. Transient receptor potential ankyrin 1 (Trpa1): forward primer, 5'-TCAAGAAGGAGAGGCTGGAA-3'; reverse primer, 5'-CCGGGTGGCTAATAGAACAA-3'. Gapdh: forward primer, 5'-AACTTTGGCATTGTGGAAGG-3'; reverse primer, 5'-ACACATTGGGGGTAGGAACA-3'. qPCR reactions were performed using the following protocols. For the SYBR green PCR system, $50^{\circ} \mathrm{C}$ for $2 \mathrm{~min}, 95^{\circ} \mathrm{C}$ for $2 \mathrm{~min}, 45$ cycles of $95^{\circ} \mathrm{C}$ for $15 \mathrm{~s}$ and $60^{\circ} \mathrm{C}$ for $1 \mathrm{~min}$, and dissociation step $\left(95^{\circ} \mathrm{C}\right.$ for $15 \mathrm{~s}, 60^{\circ} \mathrm{C}$ for $20 \mathrm{~s}$, and $95^{\circ} \mathrm{C}$ for $15 \mathrm{~s}$ ). The delta-delta method was used for relative quantification of expression (6). The mRNA expression of Gapdh was used as an internal control.

Statistical analysis. The results are expressed as the mean \pm standard error of the mean (SE). All statistical analyses were conducted using GraphPad Prism 6 software (GraphPad Software, CA, USA). The two bottle tests for capsaicin and catechin were compared between the young and old mice using two-way repeated-measures analysis of variance (ANOVA) followed by Bonferroni post-hoc test. The other assays were compared between the young and old mice using Welch's $t$-test. For all analyses, differences with $p$-values less than 0.05 

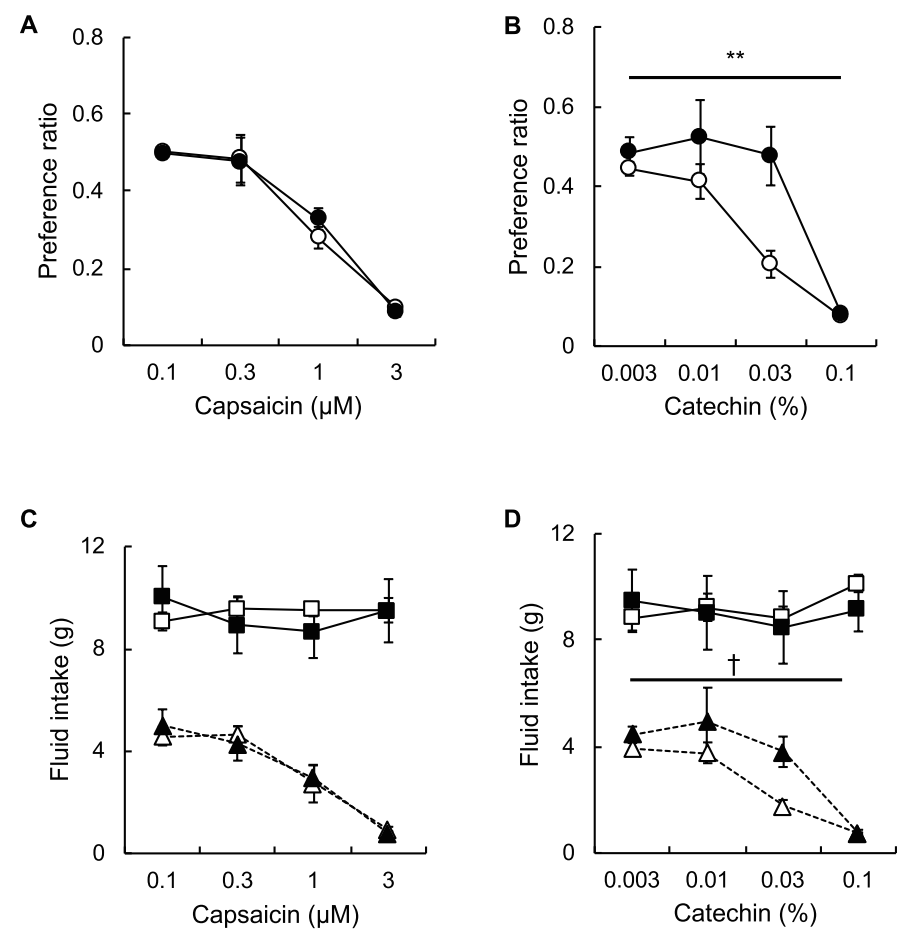

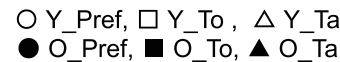

Fig. 2. Comparison of taste preferences to capsaicin and catechin between young and old mice. The preference ratios for (A) capsaicin and (B) catechin. The fluid intakes for (C) capsaicin and (D) catechin. White and black symbols are young $(n=6)$ and old mice ( $n=5$ for capsaicin and $n=6$ for catechin), respectively. For young mice, preference ratio $=Y \_$Pref, total fluid intake $=\mathrm{Y} \_$To, and taste solution intake $=\mathrm{Y} \_$Ta; For old mice, preference ratio $=$O_Pref, total fluid intake $=0 \_$To, and taste solution intake $=$ O_Ta. For preference ratio, ${ }^{* *}$ indicates $p<0.01$. For taste solution intakes, ${ }^{\dagger}$ indicates $p<0.05$ (two-way repeated-measures ANOVA).

were considered significant.

\section{RESULTS}

First, we assessed the decrease in muscle strength and change in taste preference to bitter taste (denatonium), which are characteristic phenotypes of aging in B6 mice $(4,7)$. In old mice, muscle strength was significantly decreased compared to that in young mice (Fig. 1A). In addition, we confirmed that the preference ratio for $0.3 \mathrm{mM}$ denatonium was significantly increased in old mice compared to that in young mice (Fig. 1B).

Since the characteristic phenotypes of aging were observed, we next examined whether aging affects taste preferences to capsaicin and catechin. For the capsaicin stimuli, the mice showed avoidance behavior in a concentration-dependent manner. However, there were no significant differences between the young and old mice in the preference ratio for capsaicin (Fig. 2A; $F_{(1,40)}=$ $0.05, p=0.82$ ). For the catechin stimuli, although both age groups showed avoidance behavior in a concentration-dependent manner, the preference ratios in old mice showed significantly higher values than those in young mice (Fig. 2B; $F_{(1,36)}=10.9, p<0.01$ ). Especially, there was drastically difference in the preference ratio to $0.03 \%$ catechin between young and old mice (0.003\%: $p>0.99$; $0.01 \%$ : $p=0.39$; $0.03 \%: p<0.001$; $0.1 \%: p>0.99$ ). Therefore, aging seems to influence the
A

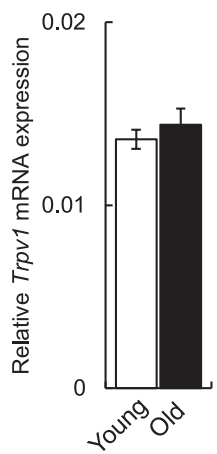

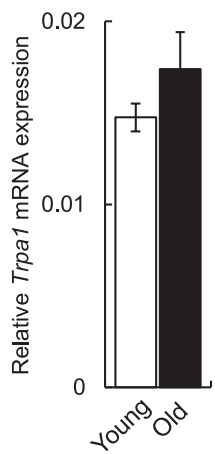

Fig. 3. mRNA expression levels of (A) Trpv1 and (B) Trpa1 in the trigeminal ganglion. White and black bars are young $(n=6)$ and old $(n=5)$ mice, respectively.

preference to catechin but not to capsaicin.

The changes in fluid intake are shown in Fig. 2C and D. There were no significantly difference in the total fluid intakes between young and old mice (Fig. 2C and D; capsaicin: $F_{(1,40)}=0.06, p=0.81$; catechin: $F_{(1,36)}=$ $0.14, p=0.71)$. Regarding the taste solutions, although there was no significantly difference in intakes of capsaicin between both groups, intakes of catechin increased significantly in old mice (Fig. 2C and D; capsaicin: $F_{(1,40)}=0.01, p=0.93$; catechin: $\left.F_{(1,36)}=6.91, p<0.05\right)$. There was a large difference in the intake of $0.03 \%$ cat- 
echin solution between both groups $(0.003 \%$ : $p>0.99$; $0.01 \%$ : $p=0.44 ; 0.03 \%$ : $p<0.05 ; 0.1 \%$ : $p>0.99)$.

Additionally, we measured expression levels of Trpv1 and Trpa1 mRNA in the TG. TRPV1 and TRPA1 are known to involve in capsaicin and catechin detection, respectively $(8,9)$. There were no significant differences in expression levels of these mRNAs between the two age groups (Fig. 3A and B), suggesting that the expression level of Trpa1 mRNA does not affect oral detection to catechin.

\section{DISCUSSION}

Physiologically, taste qualities are classified into five basic categories. Basic taste is detected by taste receptors that are expressed on taste bud cells, and their taste information is transmitted from taste bud cells to taste nerves (10). Previous reports have suggested that TRPV1 and TRPA1 are involved in the detection of pungent and astringent tastes, respectively (8, 9). TRPV1 and TRPA1 are expressed on the nerve endings of the trigeminal nerve in the oral cavity and act as multimodal receptors by perceiving noxious temperatures and chemicals (11). Therefore, pungent and astringent tastes are not classified into the basic taste categories. However, since an appropriate amount of astringency and pungency in food enriches taste, these taste qualities have an important meaning when considering the taste of food.

Several studies have investigated the correlation between aging and taste sensations in humans (12-14) and rodents (1-5). Although these studies reported inconsistent results, the findings suggest that taste sensitivity is modified by aging. In contrast, regarding the effect of aging on taste sensations, there are few available studies which tested other taste substances, in addition to basic taste-inducing substances. For example, the following compounds are used as basic taste-inducing substances: salty-sodium chloride, sourhydrochloric acid $(\mathrm{HCl})$ and citric acid, bitter-denatonium benzoate and/or quinine-HCl, sweet-sucrose and/or saccharin, and umami-monosodium glutamate, disodium inosinate, and disodium guanylate. In this study, we focused on familiar food components which are included in our daily meals, namely capsaicin and catechin, and investigated age-dependent changes in taste preferences for these substances.

Capsaicin is an alkaloid found in hot peppers and is a representative pungent component. Catechin is a representative polyphenol found in tea and wine, which induces a bitter and astringent taste. To clarify age-related changes in taste preferences for capsaicin and catechin, we compared the preference ratios to these substances between young and old mice. We observed that there was no significant difference in the preference ratio for capsaicin between young and old mice. In contrast, the preference ratio for catechin in old mice was higher than that in young mice. Moreover, old mice showed approximately 0.5 in the preference ratio of $0.03 \%$ catechin, and their total fluid intake was a similar value with the other total intakes (Fig. 2D). These suggest that catechin sensitivity decreases with age, but capsaicin sensitivity does not.

We used a commercial catechin mixture as the test catechin. EGCg is the major component (46\%) in the sample. EC, ECg, EGC, and EGCg are known to be major catechin in green tea. The taste features of these catechin obtained from a human sensory study are described below (15): ECg shows the strongest taste intensity, followed by EGCg; taste intensities of EC and $\mathrm{EGC}$ are weaker than those of $\mathrm{ECg}$ and $\mathrm{EGCg}$; they possess a complex taste that consists primarily of bitterness and astringency; and bitterness is stronger than astringency when tasting EGCg. Although astringency is interpreted as a taste separate from the sensation of bitternesss (16), the details differentiating these two sensations remain unclear. We previously reported that old B6 mice show a decrease in avoidance of bitter substances $(4,7)$. Moreover, it has been reported that ECg and EGCg are capable of activating bitter taste receptors in mice (17). These data suggest that the bitterness of catechin contributes to an observed decrease in avoidance behavior (Fig. 2B). In future we plan to investigate the effect aging has on the detection of the different catechins because each of them represents a different taste feature. Notably, the catechin mixture contained caffeine $(0.8 \%)$. As B6 mice show avoidance behavior towards $>1 \mathrm{mM}$ caffeine (18), we consider that the caffeine present in our catechin solutions (approximately 1 to $40 \mu \mathrm{M}$ ) did not have a significant effect on the avoidance behavior shown towards them.

We previously compared the peripheral taste detection systems between young and old mice to investigate the contributing factors associated with age-dependent changes in taste sensitivity $(4,5)$. Although age-associated changes in taste sensitivity were noted, age-related degradation of the peripheral taste organs, such as the expression levels of taste-related molecules and the turnover rate of taste bud cells, have yet to be confirmed. We concluded that aging-related changes in taste sensitivity may cause by factors other than those responsible for the deterioration of taste detection systems in the oral cavity $(4,5)$. It has been reported in the literature that capsaicin activates TRPV1 (8). Moreover, it has also been suggested TRPA1 (9) and the bitter taste receptor $(17,19,20)$ are involved in catechin detection. As mentioned above, we have already reported that aging did not affect the expression levels of taste-related molecules, including bitter taste receptors. Since TRPV1 and TRPA1 have been reported to be expressed on the nerve endings of the trigeminal nerve in the oral cavity (11), we confirmed age-dependent changes in the expression levels of Trpv1 and Trpa1 mRNA in the TG. However, there were no significant changes in mRNA expression levels between the two age groups (Fig. 3). This result concurs with our previous reports and suggests that expression levels of specific receptors may not be the cause of aging-related changes to taste preferences.

In this study, we confirmed the decline in catechin sensitivity due to aging. In general, it has been thought 
that taste qualities which organisms avoid are a signal of caution (e.g., bitter taste may signal the presence of poisons or toxins in food). Thus, a decrease in taste sensitivity may mean an increased risk of ingesting substances that are potentially harmful to the body. Our findings are based on the analysis of mice, and therefore, it may be difficult to extrapolate them to humans. However, when an elderly person ingests unpleasant tasting functional food components, such as catechin, a low taste sensitivity may be an advantage.

In our previous report using B6 mice, we observed that taste sensitivities to bitter and salty taste among the five basic taste are changed by aging $(4,7)$. This suggests that, in the basic taste, some are easily affected by aging and others are not. Thus, taste signal pathways may be selectively affected by aging. In this study, we observed the selective effect of aging on capsaicin and catechin detections (Fig. 2A and B). Iannilli et al. reported that age-related changes in taste functions are associated with changes in neuronal circuits (21). Therefore, brain function could be targeted to elucidate the molecular mechanisms underlying aging-dependent changes in taste sensitivity. However, we expect that our results will contribute useful information for clarifying the underlying mechanisms of aging-dependent changes in taste sensitivity.

\section{Authorship}

Research conception and design: MN and TM; experiments: MN; statistical analysis of the data: MN; interpretation of the data: MN and TM; writing of the manuscript: MN and TM.

\section{Disclosure of state of COI}

The authors have declared that no competing interests exist.

\section{Acknowledgments}

This work was supported in part by a Grant-in-Aid for Challenging Exploratory Research 26660106 and Scientific Research (B) 19 H02905 (MN) from the Japan Society for the Promotion of Science, by the Adaptable and Seamless Technology transfer Program through Target-driven R\&D (A-STEP) from Japan Science and Technology Agency (Grant No. JPMJTR194F), and by the Yamazaki Spice Promotion Foundation No. 259 (MN).

\section{REFERENCES}

1) Tordoff MG. 2007. Taste solution preferences of C57BL/6J and 129X1/SvJ mice: Influence of age, sex, and diet. Chem Senses 32: 655-671.

2) Shin YK, Cong WN, Cai H, Kim W, Maudsley S, Egan JM, Martin B. 2012. Age-related changes in mouse taste bud morphology, hormone expression, and taste responsivity. J Gerontol A Biol Sci Med Sci 67: 336-344.

3) Inui-Yamamoto C, Yamamoto T, Ueda K, Nakatsuka M, Kumabe S, Inui T, Iwai Y. 2017. Taste preference changes throughout different life stages in male rats. PLoS One 12: e0181650.

4) Narukawa M, Kurokawa A, Kohta R, Misaka T. 2017.
Participation of the peripheral taste system in aging-dependent changes in taste sensitivity. Neuroscience $\mathbf{3 5 8}$ : 249-260.

5) Narukawa M, Kamiyoshihara A, Kawae M, Kohta R, Misaka T. 2018. Analysis of aging-dependent changes in taste sensitivities of the senescence-accelerated mouse SAMP1. Exp Gerontol 113: 64-73.

6) Livak KJ, Schmittgen TD. 2001. Analysis of relative gene expression data using real-time quantitative PCR and the 2(-Delta Delta C(T)) method. Methods 25: 402408.

7) Narukawa M, Kamiyoshihara A, Izu H, Fujii T, Matsubara K, Misaka T. 2020. Efficacy of long-term feeding of $\alpha$-glycerophosphocholine for aging-related phenomena in old mice. Gerontology 66: 275-285.

8) Caterina MJ, Schumacher MA, Tominaga M, Rosen TA, Levine JD, Julius D. 1997. The capsaicin receptor: a heat-activated ion channel in the pain pathway. Nature 389: $816-824$.

9) Kurogi M, Miyashita M, Emoto Y, Kubo Y, Saitoh O. 2012. Green tea polyphenol epigallocatechin gallate activates TRPA1 in an intestinal enteroendocrine cell line, STC-1. Chem Senses 37: 167-177.

10) Yarmolinsky DA, Zuker CS, Ryba NJP. 2009. Common sense about taste: from mammals to insects. Cell 139: 234-244.

11) Roper SD. 2014. TRPs in taste and chemesthesis. Handb Exp Pharmacol 223: 827-871.

12) Weiffenbach JM, Baum BJ, Burghauser R. 1982. Taste thresholds: quality specific variation with human aging. J Gerontol 37: 372-377.

13) Schiffman SS, Frey AE, Luboski JA, Foster MA, Erickson RP. 1991. Taste of glutamate salts in young and elderly subjects: role of inosine $5^{\prime}$-monophosphate and ions. Physiol Behav 49: 843-854.

14) Mojet J, Christ-Hazelhof E, Heidema J. 2001. Taste perception with age: Generic or specific losses in threshold sensitivity to the five basic tastes? Chem Senses 26: 845860.

15) Narukawa M, Kimata H, Noga C, Watanabe T. 2010. Taste characterisation of green tea catechins. Int J Food Sci Technol 45: 1579-1585.

16) Schiffman SS, Suggs MS, Sostman AL, Simon SA. 1992. Chorda tympani and lingual nerve responses to astringent compounds in rodents. Physiol Behav 51: 51-63.

17) Narukawa M, Misaka T. 2018. Tas2r125 functions as the main receptor for detecting bitterness of tea catechins in the oral cavity of mice. Biochem Biophys Res Commun 503: 2301-2305.

18) Mura E, Taruno A, Yagi M, Yokota K, Hayashi Y. 2018. Innate and acquired tolerance to bitter stimuli in mice. PLoS One 13: e0210032.

19) Narukawa M, Noga C, Ueno Y, Sato T, Misaka T, Watanabe T. 2011. Evaluation of the bitterness of green tea catechins by a cell-based assay with the human bitter taste receptor hTAS2R39. Biochem Biophys Res Commun 405: 620-625.

20) Yamazaki T, Narukawa M, Mochizuki M, Misaka T, Watanabe T. 2013. Activation of the hTAS2R14 human bitter-taste receptor by (-)-epigallocatechin gallate and (-)-epicatechin gallate. Biosci Biotechnol Biochem 77: 1981-1983.

21) Iannilli E, Broy F, Kunz S, Hummel T. 2017. Age-related changes of gustatory function depend on alteration of neuronal circuits. J Neurosci Res 95: 1927-1936. 